\title{
Towards a Parameter Tuning Approach for a Map-Matching Algorithm
}

\author{
Carola A. Blazquez, Jana Ries, and Pablo A. Miranda
}

\begin{abstract}
Map Matching Algorithms (MMA) are developed to solve spatial ambiguities that arise in the process of assigning GPS measurements onto a digital roadway network. There is a lack of systematic parameter tuning approaches for optimizing the MMA performance. Thus, a novel integrated framework is proposed for a systematic calibration of the parameters of a post-processing MMA. The calibration approach consists of an Instance-specific Parameter Tuning Strategy (IPTS) that employs Fuzzy Logic principles. The proposed fuzzy IPTS tool determines the best algorithm parameter values by using instance-specific information a priori to the execution of the MMA. A preliminary prototype of an IPTS system is designed based on real-world data, which identifies the explanatory variables that condition the MMA performance. The implementation of the fuzzy IPTS tool on real-word data yields an enhanced MMA performance in the solution quality and computational time compared to the results of the execution of the MMA with constant algorithm settings.
\end{abstract}

\section{INTRODUCTION}

Global Positioning Systems (GPS) technology is commonly employed to acquire positioning data. The integration of GPS with Geographic Information Systems (GIS) is part of the innovative advanced technology applied by Intelligent Transportation Systems (ITS) to improve mobility and safety, reduce environmental impacts and energy consumption, and enhance the overall quality of life of individuals. When integrating GPS measurements with the roadway centerline network in GIS, these measurements (represented as data points) are usually orthogonally projected to the nearest roadway centerline segment to determine the road on which events and incidents occur, point features are located, or a vehicle is traveling.

A spatial ambiguity, spatial mismatch, or map-matching problem arises when GPS measurements are incorrectly assigned to the roadway segment due to roadway network complexities, inadequate GPS data collection procedures, lack of accuracy in the digital roadway map and the GPS measurements, inadequate sampling rate, or combinations of them $[1,2,3]$. Therefore, objects may appear to be located or moving along incorrect roadway centerlines particularly at complex roadway structures such as ramps, divided highways, overpasses and underpasses, and intersections. The mapmatching problem may arise dynamically in different instants of time such as with each GPS measurement.

* Research supported by project funds at Universidad Andres Bello Project $\mathrm{N}^{\circ}$ DI-1236-16/RG, and the Fondecyt Project $\mathrm{N}^{\circ} 1140811$.

C. Blazquez is an associate professor at Universidad Andres Bello, Viña del Mar, Chile (corresponding author to provide phone: +56 32284 5579; email: cblazquez@unab.cl.
As a result, this problem may influence negatively any subsequent usage, evaluation, analysis, planning, or decisionmaking. The distinct need for reducing incidents (accidents), travel time, congestion, and costs, while increasing customer service, productivity, efficiency, and safety in numerous ITS applications such as vehicle navigation and route guidance, fleet management, and accident prevention are only some of the major motivations for solving the map-matching problem by determining correct vehicle or user locations.

Map Matching Algorithms (MMAs) are implemented to solve the map-matching problem that occurs in different transportation applications. These MMAs employ control or weight parameters. Success in resolving the map-matching problem depends on the values assigned to each control parameter of these algorithms. The quality and geometry of the spatial data (e.g., GPS measurements, roadway centerline map, or both) and sampling frequency (e.g., every 2 seconds or 5 minutes) impact the appropriateness of values for these parameters and, thus, the performance of MMAs. Traditionally, the parameter values are established for data collected in specific environment settings such as dense urban areas or rural [4]. Hence, the reported performance and parameters values of MMAs may be conditioned and biased according to the dataset employed. In addition, scarce literature exists on methodologies to determine parameter values for optimizing the performance of MMAs. For example, Velaga et al [5] minimized the map-matching error to determine optimal weight coefficients for data collected in rural areas. Oran and Jaillet [6] developed precise proximity weight formulation for any type of MMAs.

Therefore, the objective is to develop a parameter calibration approach based on fuzzy logic principles for a topological post-processing MMA developed by Blazquez et al. [7]. The proposed parameter setting approach addresses the lack of systematic tuning techniques in the field of MMAs, and the understanding of the MMA performance behavior as a function of the environment settings or data characteristics.

\section{BACKGROUND AND LITERATURE REVIEW}

\section{A. MMAs and Transportation Applications}

MMAs have been widely studied for decades by employing different levels of complexity ranging from simple geometrical techniques to complex, advanced inference, filtering, and mathematical modeling methodologies such as

J. Ries is a Senior Lecturer at the University of Portsmouth, UK, e-mail: Jana.Ries@port.ac.uk

P. Miranda is a full time faculty member at the School of Industrial Engineering at the Pontificia Universidad Catolica de Valparaiso. e-mail: pablo.miranda@pucv.cl 
Kalman filters, Fuzzy Logic, particle filtering, hidden Markov models, and Bayesian statistics [2, 7, 8, 9, 10, 11, 12]. Topological network-based MMAs have been used particularly in ITS applications due to their simplicity, efficiency, rapid, and easy implementation [7, 8, 9, 12]. These MMAs rely on the computation of shortest paths between pairs of matched GPS points using roadway network topology (i.e., link connectivity and contiguity) and turn restrictions. As opposed to advanced MMAs, which are complex and computationally intensive, topological MMAs provide positions as accurate as possible without the need of large memories $[7,8,13,14,15,16]$.

Post-processing MMAs determine the correct road on which a user or vehicle is traveling once the complete or part of the GPS dataset is available. In this type of MMA, a large amount of data is processed to correctly determine the complete path of a user or vehicle $[4,9]$. For example, postprocessing MMAs are needed to obtain correct paths when collecting multi-modal trip data with GPS receivers for route choice modeling in a GIS environment [1, 14, 17, 18, 19]. Blazquez and Vonderohe [2] employed a topological MMA in post-processing mode to determine the correct roadway centerline on which a fleet of winter vehicles has traveled to compute winter maintenance performance measures for decision-makers and planners. Automatic road toll system based on the total mileage driven per vehicle equipped with GPS technology is another example that requires postprocessing MMAs to prevent errors in road use charging [20]. Hence, the execution of MMAs in post-processing mode has been widely studied and implemented in transportation and logistics applications [21].

\section{B. Topological Map Matching Algorithm}

The post-processing MMA employed in this study resolves the map-matching problem by determining the correct roadway centerline on which a vehicle is traveling. First, this MMA selects all roadway centerlines within a buffer around each analyzed GPS data point and orthogonally projects it to the closest roadway centerline (also known as "snapping"). Subsequently, Dijkstra's algorithm is employed to solve the shortest path problem between previously snapped and current snapped points using network topology and turn restrictions. A path is considered viable and snapped data point locations are accepted, if the difference between the computed travel speed and the average recorded vehicle speed for the data points is within a speed range tolerance. If a path is rejected, data points are snapped to alternative roadway centerlines contained within their buffers, shortest paths are recalculated, and speeds are compared once again. If no other roadway centerline candidates exist within the buffers or no viable paths are obtained, then the algorithm tests for feasible paths between preceding and subsequent data points [13]. Note that this algorithm assigns a distance of zero and maintains the last vehicle position when the vehicle speed is zero. This study utilizes the main algorithm-specific parameters of the postprocessing MMA (i.e., buffer size and speed range tolerance).

Fig. 1 shows the flow diagram of the MMA with the step sequence design. First, the MMA computes the shortest path between pairs of snapped GPS data points $\left(K_{i}\right.$ and $\left.K_{j}\right)$. If this path is not feasible, then the algorithm obtains alternative roadway centerline candidates within the buffer around data point $K_{i}$. Subsequently, feasible paths are tested between the newly snapped point $K_{i}$ and the immediate neighboring points $K_{i-1}$ and $K_{j}$. If no viable paths are found, then alternative roadway centerline candidates within the buffer around data point $K_{j}$ are obtained, and shortest paths are recalculated and speeds are compared. A dummy Boolean variable helps to advance the range of consecutive points either backward or forward until the map-matching problem is solved or the predefined number of consecutive data points is reached.

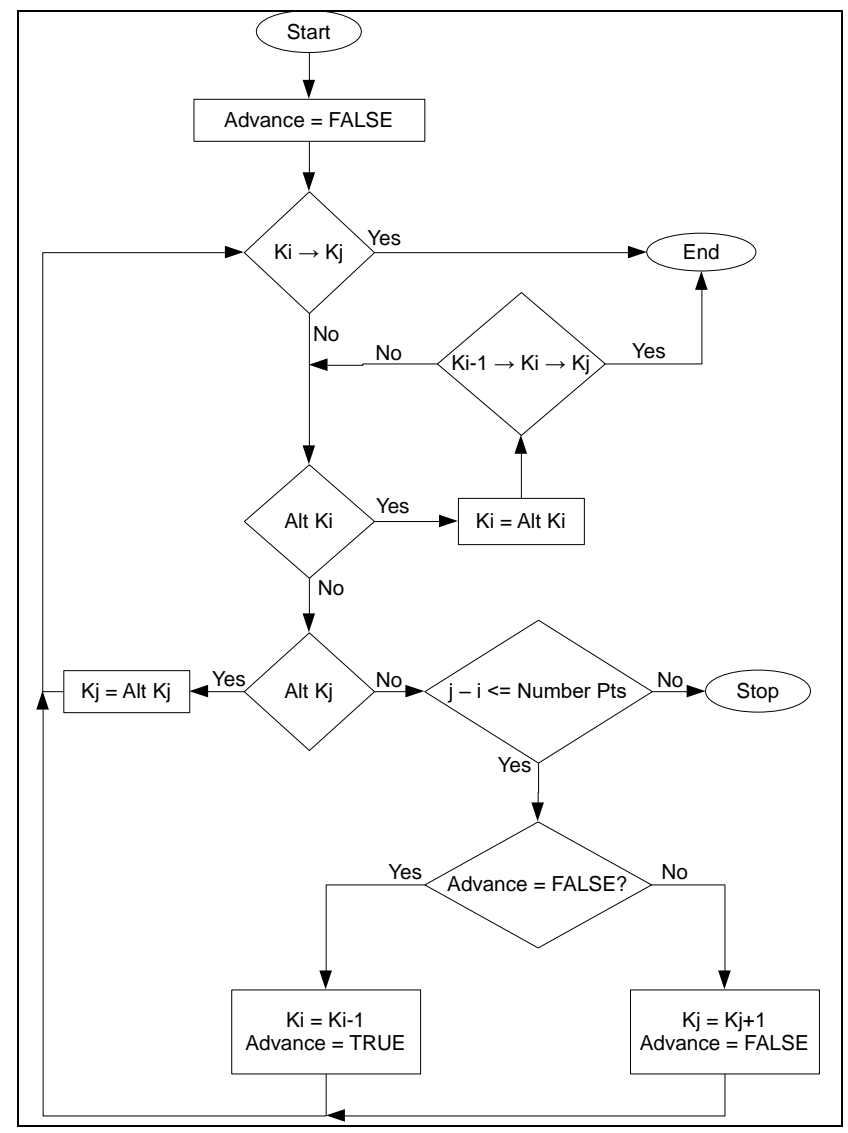

Figure 1. Flow Diagram of the post-processing MMA

\section{Parameter Setting Strategies}

Any heuristic or algorithm for a specific decisional problem performs according to the calibration of its parameters $\varphi$. This so-called parameter setting problem for an algorithm $A$ searches to determine a set of parameter values $\varphi^{*}$ that optimizes the algorithmic performance $f_{A}\left(x_{i}, \varphi\right)$, where $x_{i}$ is the problem instance under consideration. Strategies for addressing the parameter setting problem are classified into parameter control and parameter tuning, in such that the set of parameter values is integrated into the algorithm or determined a priori to executing the algorithm, respectively. Parameter control strategies commonly take the form of an adaptive strategy that allows to re-evaluate parameters $\varphi$ based on the current performance of the algorithm. Whereas, parameter tuning strategies are mainly based on a Design of Experiments (DOE), as in Coy et al. [22], Adenso-Diaz and Laguna [23], and Dobslaw [24].

Adaptive parameter control strategies require the modification of the algorithm itself, in order to integrate a reactive strategy that usually depends on the performance behavior of the algorithm. This approach is predominantly 
justified if some of the structural information of the problem instance $x_{i}$ is not known prior to executing the algorithm. This concept may be applied dynamically to adjust MMA parameters in different time instants or algorithm steps.

In contrast, parameter tuning tends to result in an average performance with its aim to provide a robust set of parameter values that can be applied to a broad variety of instances [24]. Recent studies have proposed using instance-specific information within parameter tuning strategies, so called Instance-specific Parameter Tuning Strategies, IPTS [26, 27, 28]. IPTS assesses instance-specific information a priori to the execution of an algorithm, and subsequently, determines the best set of parameter values $\varphi^{*}$ for the algorithm. An advantage of these strategies is an explicit understanding of some relevant instance-specific information of the mapmatching problem, particularly in the context of postprocessing MMAs.

\section{IPTS FRAMEWORK FOR THE POST-PROCESSING MMA}

The map-matching problem is characterized by two sets of structural information for each instance $x_{i}$, namely scenariospecific and data-specific parameters. The former consists of relevant information about the surrounding area and characteristics of a vehicle. Some of this information remains constant along the vehicle route such as type of vehicle and quality of the digital roadway network (scale and accuracy), while other information may vary as the vehicle is traveling such as maximum road speed, type of surrounding built environment (e.g., land use), weather conditions, etc. In contrast, data-specific parameters consist of information that is related to the measurements and its quality such as the number of detected satellites, vehicle speed, etc.

IPTS provide a set of parameter values $\varphi^{*}$ for one particular instance $x_{i}$ of a problem [27], which is considered to result in a good algorithmic performance. Due to the dynamic structure of the map-matching problem, this approach is extended in this paper to determine a set of best parameter values $\varphi_{r}{ }^{*}$ at a given instant or step $r$, according to the information of the instance $x_{i}\left(W_{r}\right)$. Therefore, it is important to identify instance-specific information, which is rapidly measurable and potentially has a relevant impact on the setting of algorithm-specific parameters. Note that this study analyzes two algorithm-specific parameters (buffer size and speed range tolerance), and two instance-specific parameters (sampling frequency and vehicle speed).

In order to address the parameter setting problem of the MMA, the information known for each GPS data point of an instance must be determined prior to the execution of the MMA at any step $r$. Let step $r$ be equivalent to analyzing a specific GPS data point $K_{i}$ of an instance, and step $r+1$ represents the following new data point $K_{j}$ to be examined. IPTS is called to update algorithm parameter values $\varphi_{r}{ }^{*}$ for each step $r$ independent of the changes in the instance-specific information, as shown in Fig. 2.

Zadeh [29] introduced fuzzy logic as an alternative concept to classic set theory in the case of dealing with non-crisp information. Fuzzy logic allows partial membership of information to multiple sets and is commonly applied in control strategies using a set of membership functions and a rule base to form a fuzzy system. This study proposes an IPTS tool that uses a fuzzy logic system to determine a set of algorithm parameter values based on the instance-specific information.

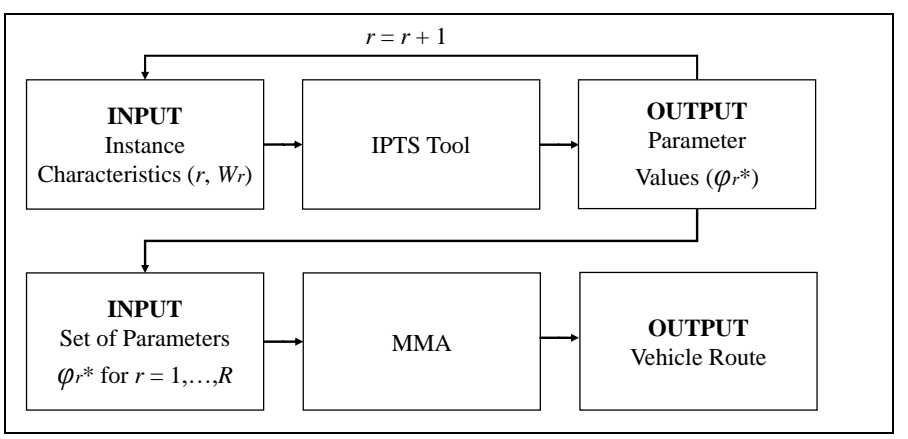

Figure 2. Diagram of the IPTS framework for the MMA

For the concept of the fuzzy IPTS tool, any instancespecific information is considered an input variable and all algorithm-specific parameters are referred to as output variables, as shown on the top part of Fig. 2. In a fuzzification stage of the fuzzy IPTS tool, instance-specific information is mapped to a set of membership functions that are associated with linguistic terms such as 'slow', 'medium' and 'fast', in order to identify a so-called membership degree $\mu$. Subsequently, a set of IF-THEN rules are activated according to the identified membership degrees (e.g., IF vehicle speed is high, THEN buffer size is medium), in order to determine the set of membership degrees for the algorithm-specific parameters. Subsequently, the fuzzy IPTS tool defuzzifies this information to derive crisp values for each output parameter of the algorithm. Finally, the outcome of the IPTS tool is a set of best parameter values $\varphi_{r} *(r=1, . ., R)$, with $R$ being the last recorded data point of the instance. The MMA employs this set of parameters to generate the complete vehicle route, as presented in the bottom part of Fig. 2.

The proposed conceptual framework requires the development of three major stages: (1) Investigating the impact of instance- and algorithm-specific information on the MMA performance, which is usually based on a factorial design or DOE; (2) Designing the fuzzy IPTS tool based on the previous stage; and (3) Executing the fuzzy IPTS tool prior to the post-processing MMA. An example of a realworld case is applied to the conceptual framework stages.

\section{RESUlts}

\section{A. Data}

The data employed in this study were collected by a winter maintenance vehicle in Portage County, Wisconsin in the United States. Three instances (routes) with a total of 60,198 GPS data points were collected along a major highways, ramps, and local roads during a winter storm. Fig. 3 presents a data sample of the collected GPS data points and roadway centerline map with a nominal scale of 1:12,000. The measured data captured coordinates, timestamp, and vehicle speeds every 10, 20, and 30 seconds (sampling frequencies). The mean, standard deviation, minimum, and maximum values of the vehicle speed data were $21.1 \mathrm{mi} / \mathrm{h}, 13.9 \mathrm{mi} / \mathrm{h}, 0$ $\mathrm{mi} / \mathrm{h}$, and $82 \mathrm{mi} / \mathrm{h}$, respectively. 


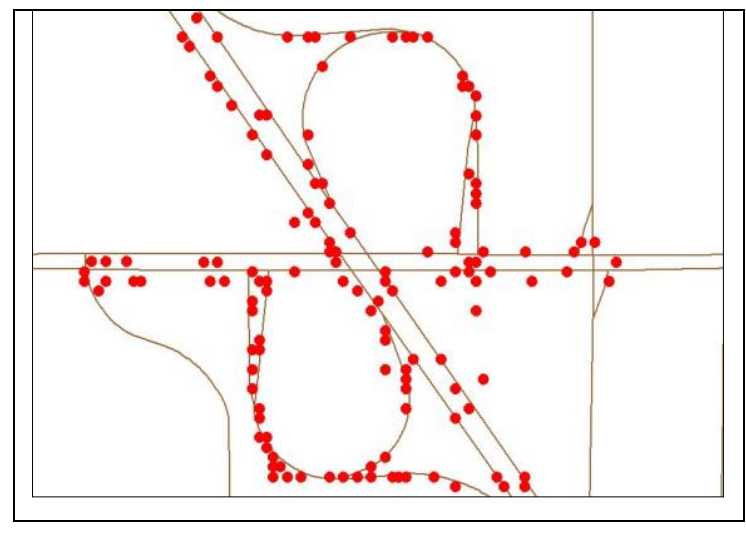

Figure 3. Data sample for Portage County

This study employed two instance-specific variables, vehicle speed and sampling frequency. Additional data such as dead reckoning and heading was not available in the data collection process. However, the set of instance-specific information can be extended by incorporating additional parameters to tailor the system to a particular context or include any other expert-based knowledge.

\section{B. Proposed Framework Stages}

\section{Stage 1: Design of Experiments (DOE)}

Stage 1 analyzes the impact of fluctuations of each parameter on the performance of the MMA using a factorial design and a regression analysis. This statistical analysis helps to comprehend the relationship between the instance characteristics and the algorithm parameters. Hence, a set of training instances were solved for different combinations of the instance-specific information taken into consideration in this study. The two instance-specific variables, vehicle speed (S) and sampling frequency (SF), are the input variables of the proposed IPTS framework. The two algorithm-specific parameters, namely buffer size (BS) and speed tolerance range (ST), are the output variables determined by the IPTS tool.

While SF, BS, and ST are variables that have been set to discrete values with three levels, $S$ is a continuous variable by nature. A preliminary clustering method was applied to categorize S. Hierarchical clustering technique was used to identify a suitable number of clusters, followed by K-means clustering to determine the associated cluster centers. These centers represent the levels of speed in the factorial design. In the tested set of instances, four clusters or levels were identified for $\mathrm{S}$ (i.e., $1.8,15,28$, and $44 \mathrm{mi} / \mathrm{h}$ ), as shown in Table I.

Due to the post-processing context of the MMA, the correct or incorrect snapping result of each data point is known in advance. The performance of the MMA was assessed by comparing the snapping result of the data point $K_{i}$ to its true route with ' 1 ' representing a correct snapping, and ' 0 ' otherwise. Table II shows the post-processing MMA performance through the percentage of correct snaps for nine combinations of constant algorithm parameter values (BS, ST) given three levels of sampling frequency (SF). Overall, the MMA performed better for data collected every 10 seconds, and as the buffer size and speed tolerance increase. Thus, the combination $(60,35)$ for all sampling frequency levels will be employed for testing the proposed framework in Stage 3.
TABLE I. FACTORIAL DESIGN

\begin{tabular}{|l|c|}
\hline \multicolumn{1}{|c|}{ Parameter } & Factorial Design Levels \\
\hline Buffer Size (BS) $[\mathrm{ft}]$ & $20,40,60$ \\
\hline Speed Tolerance (ST) $[\mathrm{mi} / \mathrm{h}]$ & $15,25,35$ \\
\hline Sampling Frequency (SF) [s] & $10,20,30$ \\
\hline Vehicle Speed (S) $[\mathrm{mi} / \mathrm{h}]$ & $1.8,15,28,44$ \\
\hline
\end{tabular}

TABLE II. MMA PERFORMANCE RESULTS

\begin{tabular}{|c|c|c|c|}
\hline \multirow{2}{*}{$\begin{array}{c}\text { Constant Parameter } \\
\text { Settings } \\
\text { (BS, ST) }[\mathrm{ft}, \mathrm{mi} / \mathrm{h}]\end{array}$} & \multicolumn{3}{|c|}{ Sampling Frequency, SF [s] } \\
\cline { 2 - 4 } & $\mathbf{1 0}$ & $\mathbf{2 0}$ & $\mathbf{3 0}$ \\
\hline$(20,15)$ & $95.8 \%$ & $94.8 \%$ & $94.0 \%$ \\
\hline$(20,25)$ & $96.3 \%$ & $95.9 \%$ & $95.1 \%$ \\
\hline$(20,35)$ & $96.5 \%$ & $95.9 \%$ & $95.3 \%$ \\
\hline$(40,15)$ & $96.9 \%$ & $96.4 \%$ & $96.2 \%$ \\
\hline$(40,25)$ & $96.9 \%$ & $96.5 \%$ & $96.2 \%$ \\
\hline$(40,35)$ & $97.0 \%$ & $96.8 \%$ & $96.7 \%$ \\
\hline$(60,15)$ & $97.8 \%$ & $97.1 \%$ & $96.8 \%$ \\
\hline$(60,25)$ & $97.9 \%$ & $97.7 \%$ & $97.3 \%$ \\
\hline$(60,35)$ & $97.9 \%$ & $97.7 \%$ & $97.3 \%$ \\
\hline
\end{tabular}

TABLE III. LOGISTIC BINARY REGRESSION RESULTS

\begin{tabular}{|l|l|l|}
\hline \multicolumn{1}{|c|}{ Variables } & \multicolumn{1}{|c|}{$\begin{array}{c}\text { Regression } \\
\text { Coefficient (b) }\end{array}$} & Odds Ratio $\boldsymbol{e}^{\mathbf{b}}$ \\
\hline Constant & $-1.429 * *$ & \\
\hline Main Effects & $0.009 *$ & 1.009 \\
\hline ST & $0.027 * *$ & 1.023 \\
\hline BS & $0.873^{*} *$ & 2.393 \\
\hline S & $-0.003 *$ & 0.995 \\
\hline $2-$ way interaction & $-0.001 * *$ & 0.999 \\
\hline ST x S & $-0.02 * *$ & 0.978 \\
\hline SF x BS &
\end{tabular}

$\mathrm{R} 2=0.126$ (Cox \& Snell), 0.169 (Nagelkerke)

Since a categorical binary dependent variable was employed in the MMA performance analysis, a binary logistic regression analysis was conducted. This regression was used to investigate potential main and 2-way interaction effects with respect to the MMA performance. In other words, the statistical analysis indicates whether the variation of one parameter, with all others being equal, leads to a statistically significant change in the performance (main effect), and whether the impact of one independent variable varies depending on the level of a second independent variable (2way interaction).

Table III shows the regression coefficients of the significant factors of the analysis, and the odds ratios, $e^{\mathrm{b}}$, for main and 2-way interaction effects. An odds ratio larger than 1 indicates that the odds of the dependent variable increases (i.e., overall MMA performance is improved) with increases in the independent variables. Conversely, if the odds ratio is lower than 1, then an increase in the independent variable decreases the odds of correct snapped data points onto the roadway centerline. The results outline that if ST, BS, or S are increased, then the odds of a correct snap increases, with the latter having the largest impact. SF did not show a statistically significant main effect. However, this variable is relevant in two interaction effects, with variables BS and S. An interesting interaction occurs between ST and S. If S is high, then there is an indication that different ST values do not make a difference. However, if $\mathrm{S}$ is low, then the results indicate that odds of a correct snap are increased by choosing higher levels of ST. Another interesting interaction effect is the combination of SF 
and BS, in which for small SF values, higher levels of BS result in increased odds of correct snaps. However, for larger $\mathrm{SF}$ values, the level of $\mathrm{S}$ becomes irrelevant, leading to an overall worse MMA performance.

\section{Stage 2: Fuzzy IPTS tool design}

The insights drawn from the regression analysis based on the factorial analysis form the basis of a manual design of a fuzzy inference system. In the fuzzification stage of the system, the factorial design mainly informs the design of the set of membership functions. Table IV shows the design of the triangular and trapezoidal membership functions for the input (instance-specific parameters) and output (algorithmspecific parameters) of the IPTS. The regression analysis was utilized to manually design a rule base for the fuzzy IPTS in combination with expert knowledge on the algorithm. Table $\mathrm{V}$ shows the corresponding rule base of the proposed fuzzy IPTS. For example, if SF is small and S is fast, then BS is medium and ST is high.

TABLE IV. MEMBERSHIP FUNCTIONS FOR INPUT AND OUTPUT

\begin{tabular}{|c|c|c|c|}
\hline \multicolumn{4}{|c|}{ INPUT } \\
\hline & Min & Medium & $\operatorname{Max}$ \\
\hline \multicolumn{4}{|c|}{ Sampling Frequency, SF [s] } \\
\hline Small & 0 & 10 & 20 \\
\hline Medium & 10 & 20 & 30 \\
\hline Large & 20 & 30 & 40 \\
\hline \multicolumn{4}{|c|}{ Speed, $\mathbf{S}[\mathrm{mi} / \mathrm{h}]$} \\
\hline Very Slow & 0 & 5 & 10 \\
\hline Slow & 5 & 15 & 20 \\
\hline Medium & 15 & 30 & 45 \\
\hline Fast & 30 & 45 & 60 \\
\hline \multicolumn{4}{|c|}{ OUTPUT } \\
\hline & Min & Medium & $\operatorname{Max}$ \\
\hline \multicolumn{4}{|c|}{ Buffer Size, BS [ft] } \\
\hline Small & 0 & 20 & 40 \\
\hline Medium & 20 & 40 & 60 \\
\hline Large & 40 & 60 & 80 \\
\hline \multicolumn{4}{|c|}{ Speed Tolerance, $\mathrm{ST}[\mathrm{mi} / \mathrm{h}]$} \\
\hline Low & 5 & 15 & 25 \\
\hline Medium & 15 & 25 & 35 \\
\hline High & 25 & 35 & 45 \\
\hline
\end{tabular}

TABLE V. RULE BASE FOR THE FUZZY IPTS

\begin{tabular}{|c|c|c|c|}
\hline \multicolumn{2}{|c|}{ INPUT } & \multicolumn{2}{c|}{ OUTPUT } \\
\hline \multicolumn{2}{|c|}{ Instance-Specific Parameters } & Algorithm-specific parameters \\
\hline $\begin{array}{c}\text { Sampling } \\
\text { Frequency }(\text { SF })\end{array}$ & Speed $(\boldsymbol{S})$ & $\begin{array}{c}\text { Buffer Size } \\
(\boldsymbol{B S})\end{array}$ & $\begin{array}{c}\text { Speed } \\
\text { Tolerance } \\
(\text { ST })\end{array}$ \\
\hline Small & Very Slow & Large & Low \\
\hline Medium & Very Slow & Small & High \\
\hline Large & Very Slow & Small & High \\
\hline Small & Slow & Large & Medium \\
\hline Medium & Slow & Small & Medium \\
\hline Large & Slow & Small & Medium \\
\hline Small & Medium & Medium & High \\
\hline Medium & Medium & Medium & Medium \\
\hline Large & Medium & Small & Medium \\
\hline Small & Fast & Medium & High \\
\hline Medium & Fast & Medium & Low \\
\hline Large & Fast & Small & Low \\
\hline
\end{tabular}

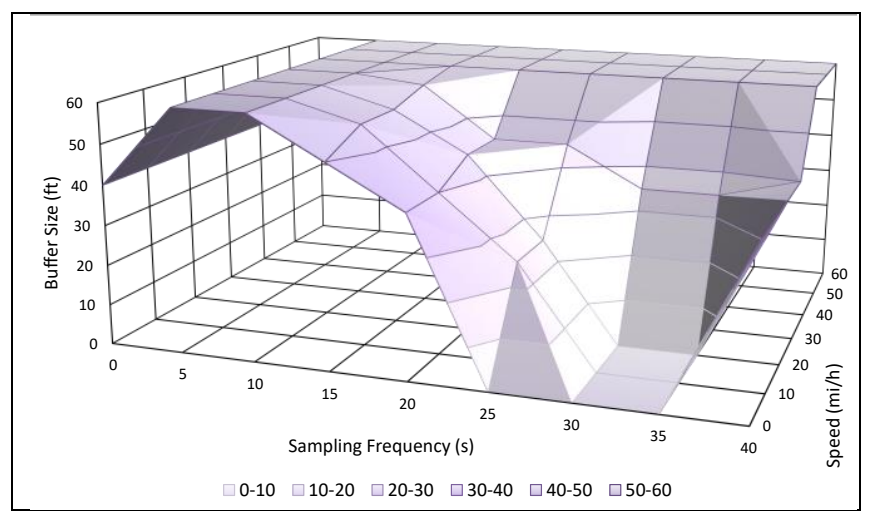

Figure 4. Surface view for Speed and Sampling Frequency on Buffer size

The fuzzy IPTS tool uses the construct of a Mamdani-type fuzzy inference system. The defuzzification process applies the centroid method, in which centers of gravity for the fuzzy sets are employed to determine crisp values. As a result of this process, the impact of different values for speed and sampling frequency on the value assigned to buffer size was obtained, as shown in Fig. 4. Note that high speed values assign large buffer sizes independent of the sampling frequency.

\section{Stage 3: Execution of Fuzzy IPTS tool prior to MMA}

The IPTS framework was applied to the test dataset with the results from the previous stage. The fuzzy IPTS tool determines the algorithm-specific parameter (BS and ST) for each data point of the test dataset a priori to the execution of the MMA. Table VI presents the results for the performance and computational time of the proposed IPTS framework and the constant MMA parameter setting (BS $=60 \mathrm{ft}$ and $\mathrm{ST}=35$ $\mathrm{mi} / \mathrm{h}$ ) with the best performance (See Table II) for 10, 20, and 30 second-sampling intervals. This table shows improvements with respect to the percentage of correct snaps and computational time. These results confirm that the execution of the IPTS tool prior to the MMA helps to select the best algorithm parameter values for each analyzed data point. Therefore, the MMA is able to obtain a good solution (i.e., correct snapping location on a roadway centerline for a data point) with less number of algorithm steps and in less time.

\section{CONCLUSIONS}

Scarce calibration methods exist for determining parameter values that successfully improve the performance of MMAs for any given dataset and environment settings. This study proposes a conceptual framework that executes an instancespecific parameter tuning strategy (IPTS) prior to running a post-processing topological MMA. Fuzzy logic is used in the IPTS design that considers imprecise information in the process of parameter tuning, which underpins its suitability in the context of map matching.

The proposed IPTS framework presents an overall improvement in the performance of a post-processing MMA by tailoring algorithm-specific parameters towards instancespecific information for each individual GPS data point. This framework presents a progress in relation to the existing manual trial-and-error techniques utilized to set MMA parameters. The IPTS framework yields enhanced results when compared to the best performed constant algorithm setting for different sampling frequencies. 
TABLE VI. PERFORMANCE RESULTS

\begin{tabular}{|c|c|c|c|c|c|c|}
\hline \multirow{3}{*}{ Performance } & \multicolumn{3}{|c|}{ Correct snaps [\%] } & \multicolumn{3}{|c|}{ Computational Time [s] } \\
\hline & \multicolumn{6}{|c|}{ Sampling Frequency, $S F$ [s] } \\
\hline & 10 & 20 & 30 & 10 & 20 & 30 \\
\hline $\begin{array}{c}\text { IPTS } \\
\text { Framework }\end{array}$ & 98.9 & 98.5 & 98.2 & 26627 & 26976 & 27120 \\
\hline $\begin{array}{c}\text { Constant } \\
\text { Parameter } \\
\text { Setting } \\
(60,35) \\
\end{array}$ & 97.9 & 97.7 & 97.3 & 37235 & 39560 & 39697 \\
\hline
\end{tabular}

Although improvements in the MMA performance are observed with the test sets employed in this study, the proposed framework should be further examined with other datasets to represent different types of transportation applications (e.g., fleet of vehicles, public transportation) and different environment settings.

In order to design the IPTS system, a manual expert-based approach is presented that uses insights from a statistical analysis, which informs the set-up of a fuzzy IPTS system. This approach may be modified using a semi-automated or automated approach allowing for less dependence on expert knowledge with respect to the algorithm [30].

Further research includes the update of the algorithm parameter values only when the instance characteristics of the data points present any variation, as opposed to calling the IPTS tool with each new data point. This should prevent executing the IPTS tool unnecessarily. Finally, the integration of the fuzzy IPTS tool and the MMA should be explored for solving the map-matching problem in real-time.

\section{REFERENCES}

[1] M. Bierlaire, J. Chen, and J. Newman, "A probabilistic map matching method for smartphone GPS data", Transportation Research Part C, vol. 26, pp. 78-98, 2013.

[2] C. Blazquez, and A. Vonderohe, "Simple Map-Matching Algorithm Applied to Intelligent Winter Maintenance Vehicle Data", Transportation Research Record: J. of the Transportation Research Record, vol. 1935, pp. 68-76, 2005.

[3] W. Chen, Z. Li, M. Yu, and Y. Chen, "Effects of sensor errors on the performance of map-matching", J. of Navigation, vol. 58, pp. 273282,2005

[4] R. Dalumpines, and D. Scott, "GIS-Based map-matching: development and demonstration of a post-processing map-matching algorithm for transportation research", In Advancing geoinformation science for a changing world. Lecture Notes in Geoinformation and Cartography, S. Geertman, W. Reinhardt, and F. Toppen, Ed. Berlin Heidelberg: Springer-Verlag, 2011.

[5] N. Velaga, M. Quddus, and A. Bristow, "Developing an Enhanced Weight-Based Topological Map-Matching Algorithm for Intelligent Transport Systems", Transportation Research Part C, vol.17, no. 6, pp. 672-683, 2009.

[6] A. Oran and P. Jaillet, "A Precise Proximity-Weight Formulation for Map Matching Algorithms", $10^{\text {th }}$ Workshop on Positioning, Navigation and Communication (WPNC), 2013, pp. 1-6.

[7] C. Blazquez, P. Miranda, and A. Ponce, "Performance of a New Enhanced Topological Decision Rule Map Matching Algorithm for Transportation Applications", J. of Applied Research \& Technology, vol. 10, pp. 929-940, 2012.

[8] C. Blazquez, “A Decision-Rule Topological Map-Matching Algorithm with Multiple Spatial Data", In Global Navigation Satellite Systems: Signal, Theory and Applications, J. Shuanggen, Ed. Croatia: Intechweb, 2012, pp. 215-240.

[9] M. Hashemi, and H. Karimi, "A Critical Review of Real-Time MapMatching Algorithms: Current Issues and Future Directions", Computers, Environment and Urban Systems, vol. 48, pp. 153-165, 2014.
[10] G. Taylor, and G. Blewitt, Intelligent Positioning GIS-GPS Unification West Sussex: John Wiley \& Sons, Ltd., 2006.

[11] M. Ren, and H. Karimi, "A hidden Markov model-based mapmatching algorithm for wheelchair navigation", J. of Navigation, vol. 62, no. 3, pp. 383-395, 2009.

[12] Y. Zhao, Vehicle location and navigation systems, Norwood, MA: Artech House, Inc., 1997.

[13] C. Blazquez, and A. Vonderohe, "Effects of Controlling Parameters on Performance of a Decision-Rule Map-Matching Algorithm", ASCE J. of Transportation Engineering, vol. 135, no. 12, pp. 966-973, 2009.

[14] F. Marchal, J. Hackney, and K. Axhausen, "Efficient map matching of large global positioning system data sets", J. of the Transportation Research Board, vol. 1935, no. 1, pp. 93-100, 2005.

[15] M. Quddus, W. Ochieng, and R. Noland, "Current Map-Matching Algorithms for Transport Applications: State-Of-The Art and Future Research Directions“", Transportation Research Part C, vol. 15, pp. 312-328, 2007

[16] Z. Wang, and Z. Yang, Z. "Research on the Map Matching of Typical Region Based on the Topological Analysis" in Proc. 2nd International Conference on Intelligent Computation Technology and Automation, Zhangiajie, China, 2009.

[17] D. Papinski, D. Scott, and S. Doherty, "Exploring the Route Choice Decision-Making Process: A Comparison of Planned and Observed Routes Obtained Using Person-Based GPS", Transportation Research Part F, vol. 12, pp. 347-358, 2009.

[18] T. Rasmussen, J. Ingvardson, K. Halldorsdottir, and O. Nielsen, "Improved methods to deduct trip legs and mode from travel surveys using wearable GPS devices: A case study from the Greater Copenhagen area", Computers, Environment and Urban Systems, vol. 54, pp. 301-313, 2015

[19] L. Shen, and R. Stopher, "Review of GPS Travel Survey and GPS data-processing methods", Transport Reviews, vol. 34, no. 3, pp. 316334, 2014.

[20] K. Sheridan, T. Dyjas, C. Botteron, J. Leclere, F. Dominic, and G. Marucco, "Demands of Roads. An Assisted-GNSS Solution Uses the EGNOS Data Access Service", GPS World, vol. 22, no. 3, pp. 28, 2011

[21] C. Blazquez, and P. Miranda, "A real-time topological map matching methodology for GPS/GIS based travel behavior studies", In Mobile technologies for activity-travel data collection and analysis, S. Rasouli, and H. Timmermans, Ed. New York; IGI, 2014, pp.155-173.

[22] S. P. Coy, B. L. Golden, G.C. Runger, E.C. Wasil, "Using experimental design to find effective parameter settings for heuristics", J. of Heuristics, vol.7, no. 1, pp. 77-97, 2001.

[23] B. Adenso-Diaz, and M. Laguna, "Fine-tuning of algorithms using fractional experimental designs and local search", Operations Research, vol. 54, no. 1, pp. 99-114, 2006.

[24] F. Dobslaw, "A parameter tuning framework for metaheuristics based on design of experiments and artificial neural networks" In Proc. International Conf. on Computer Mathematics and Natural Computing, 2010, pp. 1-4.

[25] M. Kern, "Parameter Adaption in Heuristic Search - A PopulationBased Approach", Ph.D. Thesis, University of Essex, UK, 2006.

[26] J. Ries, P. Beullens, and D. Salt, "Instance-specific multi-objective parameter tuning based on fuzzy logic", European J. of Operational Research, vol. 218, pp. 305-315, 2012.

[27] J. Ries, and P. Beullens, "A semi-automated design of instance-based fuzzy parameter tuning for metaheuristics based on decision tree induction", J. of the Operational Research Society, vol. 66, no. 5, pp. 782-793, 2015

[28] R. Pavon, F. Diaz, R. Laza, and V. Luzon, "Automatic parameter tuning with a Bayesian case-based reasoning system", Expert Systems with Application, vol. 36,no. 2, pp. 3407-3420, 2009.

[29] L.A. Zadeh, "Fuzzy sets", Information and Control, vol. 8, pp. 338353, 1965.

[30] J. Ries, P. Beullens, and Y. Wang, "Instance-specific parameter tuning for meta-heuristics", Meta-heuristics optimization algorithms in engineering, business, economics, and finance, T. Vasant, $\mathrm{Ed}$. Hershey: IGI Global, 2012, pp. 136-170. 